\title{
IMAGEN, CONFIANZA Y LIDERAZGO EN VOCEROS PÚBLICOS DURANTE LA PANDEMIA COVID-19. CASO: NUEVO LEÓN, MÉXICO
}

\author{
IMAGE, TRUST AND LEADERSHIP IN PUBLIC SPOKESPEOPLE DURING THE \\ COVID-19 PANDEMIC. CASE: NUEVO LEON, MEXICO
}

\author{
Felipe MARAÑÓN ${ }^{1}$ \\ Rodolfo BARRIENTOS ${ }^{2}$ \\ Luis Gerardo FRÍAS ${ }^{3}$
}

\begin{tabular}{|lll|}
\hline Recibido & $:$ & 19.04 .2021 \\
Aceptado & $:$ & 08.07 .2021 \\
Publicado & $:$ & 02.08 .2021 \\
\hline
\end{tabular}

RESUMEN: El liderazgo es un concepto difícil de definir, pero en tiempos de crisis es fácil de identificar. Para ello, la imagen y la confianza son dos características importantes que sirven para distinguir a un líder. La diversidad de contenido mediático que se puede apreciar en la actualidad y sobre todo respecto a la pandemia, genera la oportunidad de escuchar más voces e identificar a los líderes que son referencia para los ciudadanos. El objetivo principal de esta investigación cualitativa es identificar la manera en que los ciudadanos perciben la imagen, confianza y liderazgo de los voceros públicos de la pandemia Covid-19. Debido al corte cualitativo del estudio se realizaron cuatro grupos focales divididos por sexo y edad. Entre los hallazgos más importantes se encuentra que existe una mayor confianza y aceptación hacia la imagen de los portavoces del área de la salud por encima de la de los líderes políticos, quienes presentan poca confianza y una imagen controvertida.

Palabras clave: Covid-19, Imagen, Liderazgo, Medios de comunicación, Confianza.

\begin{abstract}
Leadership is a difficult concept to define, but in times of crisis it is easy to identify. For this, image and trust are two important characteristics that are useful to distinguish a leader. The diversity of media content that can be seen today and especially with regard to the pandemic, generates the opportunity to hear more voices and identify leaders that are a reference for citizens. The aim of this qualitative research is to identify the way in which citizens perceive the image, trust and leadership in public spokespeople of the COVID-19 pandemic. Due to the qualitative nature of this study, four focus groups were conducted, divided by sex and age. Among the most important findings is that there is a greater trust and acceptance towards the image of the spokespeople of the health area over that of political leaders, who had little trust and a controversial image.
\end{abstract}

Keywords: COVID-19, Image, Leadership, Mass Media, Trust.

\footnotetext{
${ }^{1}$ Doctor en Filosofía con orientación en Ciencias Políticas, Universidad Autónoma de Nuevo León - Facultas de Ciencias Políticas y Relaciones Internacionales, Monterrey, Nuevo León. México. Email: felipe.maranonlzcn@uanl.edu.mx. ORCID: https://orcid.org/0000-0002-0705-6336

${ }^{2}$ Doctor en Filosofía con orientación en Ciencias Políticas. Universidad Autónoma de Nuevo León - Facultas de Ciencias Políticas y Relaciones Internacionales, Monterrey, Nuevo León. México. Email: rodolfo.barrientosu@ gmail.com. ORCID: https://orcid.org/0000-0003-0460-5974

${ }^{3}$ Doctor en Estudios Humanísticos en la línea de investigación de Comunicación y Estudios culturales. Instituto Tecnológico y de Estudios Superiores de Monterrey. Monterrey, Nuevo León. México. Email: luisgerardofrias@ hotmail.com. ORCID: https://orcid.org/0000-0002-9363-1543
} 


\section{Journal of the Academy $|8|$}

\section{INTRODUCCIÓN}

El Covid-19 se ha convertido en una situación complicada como lo aseveran Kleinberg et al., (2020) al observar un impacto dramático en la sociedad y en las economías alrededor del mundo, en respuesta a las emociones de las personas. Por lo tanto, es importante entender la forma en que se perciben las notas y contenidos mediáticos de la pandemia, ya que son los principales transmisores de conocimiento sobre el acontecer diario a los ciudadanos (Califano, 2015).

$\mathrm{Al}$ analizar constructos como la imagen, el liderazgo y la confianza de quienes informan sobre la salud, en este caso específico sobre el Covid-19, se observa de forma más completa la percepción de la gente acerca del contenido informativo, donde se busca la comprensión de mensajes efectivos en materia de salud (Malecki et al., 2020). Por lo que la comunicación efectiva sobre los riesgos que se presentan frente las pandemias debe abordarse de manera rápida y buscando estrategias que favorezcan su pronta comprensión como lo afirma Glik (2007), "los desafíos frente a una pandemia se centran en la comunicación de estrategias para abordar los desafíos en salud pública” (p. 2).

Actualmente los expertos utilizan medios de comunicación para la difusión de peligros que se generan en la presente crisis sanitaria, controlando y ayudando a mitigar el miedo y la desesperación social, convirtiéndose así en una de las fuentes más confiables ante la pandemia (Malecki et al., 2020). Es así que, por medio de la aplicación de grupos focales, la presente investigación buscó identificar la percepción y comprensión que tienen los ciudadanos sobre la confianza, imagen y el liderazgo que los medios de comunicación y agentes públicos transmiten para informar durante la presente crisis sanitaria.

El objetivo de la presente investigación fue identificar la manera en que los ciudadanos perciben la imagen, confianza y liderazgo de los voceros públicos de la pandemia Covid-19 que se presenta a través de los medios de comunicación en México, en específico en el estado de Nuevo León. 


\section{Journal of the Academy $|9|$}

\section{DESARROLLO}

\section{Los voceros públicos en las crisis sociales: imagen y comunicación}

Los medios de comunicación han desempeñado un papel fundamental en la sociedad, ya que la información de interés público se emite por medio de estos. La información política ha sido emitida durante años por medio de la televisión, siendo este medio el más confiable por el hecho de registrar los acontecimientos políticos visualmente. Esta misma comunicación ha sido de vital importancia en la sociedad ya que "la comunicación sirve para orientar a la sociedad por medio de la definición de unos objetivos y de la identificación de problemas" (Canel, 2006, p. 19). De esta forma la comunicación se presenta como un puente entre ciudadano y estado, generando una mejor interacción entre ellos.

La comunicación es diferente durante una situación de crisis o de emergencia, en este contexto los medios de información estarán involucrados en reportar la situación de manera oportuna, transparente y fidedigna generando una imagen confiable de quien tenga el liderazgo para ser portavoz durante una situación de riesgo, es por ello por lo que surge la imagen del vocero público $(P A H O, 2010)$. Para llevar a cabo dicho evento, el vocero ante la situación de emergencia debe incluir la información nueva que le quiera transmitir al público y presentar una imagen agradable y que genere confianza ya sea por medio de su manera de hablar, vestir o moverse (Martín-Salgado, 2002).

Montaña, et al. (2020) indican que, en tiempos de crisis y pandemia, como sucede actualmente con el Covid-19, el consumo de medios tanto tradicionales como online aumenta en gran medida debido al confinamiento que solicitan tanto gobierno como sector salud. Por lo tanto, la información sobre lo que acontece diariamente respecto a este tema se recibe por parte de voceros oficiales, quienes se vuelven justamente la fuente de información oficial para el ciudadano. Por su parte, Rodero (2020) menciona que la televisión y el radio se erigen como los medios que mayor confianza dan a las personas para informarse, no solamente en las versiones tradicionales de ambos medios, sino también en las digitales.

La Organización Panamericana de la Salud (2010), PAHO por sus siglas en inglés, indica que uno de los requisitos básicos para la imagen de un vocero exitoso es poder comunicarse de 


\section{Journal of the Academy $|10|$}

manera efectiva con los demás y entender bien la situación y el papel que representan. Los voceros pueden reducir la ansiedad y el temor brindado a las comunidades la información adecuada para cuidar y recuperar la salud durante una pandemia. Es importante tomar en cuenta que no son solo las palabras que comunican, sino también el modo como se expresa y la manera en que es visto por los demás. De esta manera, la imagen de la vocera o vocero en cuestión cobrará mayor relevancia por lo que, tanto su figura como sus atributos, tomarán igual importancia que el discurso dictado. Así las gesticulaciones, accesorios, símbolos, actitudes y comportamientos, en lo público y lo privado, pasarán a ser parte de la personalización de este vocero público. (Orejuela, 2009).

La imagen se crea cuando la apariencia simboliza una realidad, en este caso, la imagen del personaje político es un interés social que se transforma en un mensaje directo para los ciudadanos, por tanto, esta imagen se integra a un proceso comunicativo, por lo que es fundamental distinguir entre la imagen proyectada y la imagen que percibe el ciudadano (Orejuela, 2009). Al ser parte de la comunicación social, Martín-Salgado (2002) explica que la imagen proyectada está relacionada con el carisma, que designa unas características peculiares del individuo dándole una personalidad especial; lo cual influye en el modo de elaborar un mensaje y, por consiguiente, puede ser algo extrínseco al personaje mismo y llega a ser ajena y con un bajo nivel de asociación. En cambio, la imagen percibida es la transmitida y filtrada referente al nivel de conocimiento y de información del personaje.

En un estudio de Miller et al., (1986) las cinco valoraciones positivas que encuentran para describir una buena imagen, son: 1) la competencia, es decir, el intelecto y experiencia laboral de la persona; 2) la integridad, en los que el personaje demuestra o expresa valores como la honestidad y confianza; 3) el ser fiable, valores como el compromiso y la entrega a la labor; 4) el carisma, la forma en que se comunica y conecta con las personas, incluidas a las que dirige; y, por último, 5) la apariencia física o rasgos sociodemográficos como: edad, habilidades retóricas, religión, etc.

Por su parte, Canel (2001) agrupa las constantes de una imagen positiva en cinco clases: características biográficas, características personales, cualificación profesional, posicionamiento ideológico y habilidades comunicativas. Cada una de las categorías están comprendidas por diferentes ítems según corresponda, es decir, las características biográficas 


\section{Journal of the Academy $|11|$}

comprenden la edad, sexo, educación, nivel cultural, creencias y vida familiar; por otro lado, las características personales abarcan la simpatía, la impulsividad, la sinceridad, la madurez, la integridad y la manera en que realiza sus actividades.

Mientras tanto, la cualificación profesional se refiere a currículo, experiencia de gobierno y visión de conjunto; la categoría de posicionamiento ideológico está constituida por ítems como las aspiraciones políticas, militancia de grupos de interés y la afinidad con los ideales de los partidos políticos (Canel, 2001). Por último, las habilidades comunicativas las conforman la telegénica, la oratoria, la agilidad de argumentación, la claridad de expresión, timbre de voz y la gesticulación, etcétera (Puig, 2009; Chihu, 2010; Gómez, 2001). De esta manera, se encuentra una serie de características físicas, biológicas, sociales y actitudinales que permiten analizar la personalización de personajes políticos relevantes, en este caso, a las voceras o voceros durante una crisis sanitaria como la del Covid-19.

\section{La confianza del ciudadano a los servidores públicos}

Es evidente la necesidad de que tanto los procesos electorales como la llegada al poder de los representantes políticos estén envueltos en un ambiente de confianza y transparencia. Sobre todo, cuando la confianza y la credibilidad son indicadores básicos de la legitimidad, entendida como la creencia en que las instituciones políticas son las mejores para la sociedad (Fabián, 2011). El término confianza se aborda en autores clásicos como Hobbes, Locke, Tocqueville, Weber o Parsons y aunque es difícil de definir, autores como Landau (2009) y Güemes (2016) arrojan una definición que aborda la relación entre lo que espera un individuo sobre lo que otras personas en teoría realizarían en esta visión positiva del individuo inicial. Sin embargo, ya sean individuos u organizaciones, este sentimiento de confianza se transforma en decepción cuando se llega a un estado intermedio entre el saber y el no saber.

Aunado a la confianza, la eficacia política de los ciudadanos ha sido limitada por algunos autores como Benedicto y Morán (2014) quienes afirman que los ciudadanos se mantienen informados en temas que son de su interés o afecta a sus vidas, por lo tanto, representan un mosaico, una gran diversidad de ideas, públicos y competencias. En cuanto a la relación de confianza y eficacia, Beramendi, et al. (2016) establecen que la confianza política se logra con base en la eficiencia y efectividad que la sociedad percibe de las instituciones. Los ciudadanos se sienten más seguros en sus interacciones con el gobierno cuando las instituciones brindan certeza jurídica, justicia y políticas de equidad. Es así que la calidad en la gestión de los 


\section{Journal of the Academy | 12 |}

gobiernos determinará el nivel de confianza que los ciudadanos tienen hacia su gobierno (Hiskey y Seligson, 2003).

\section{El liderazgo en tiempos de pandemia}

Existen diversas acepciones sobre el término liderazgo, en las que se menciona la relación de influencia que surge por medio de la interacción entre líderes y seguidores en la búsqueda de cambios y resultados sobre determinada situación, así como la cualidad de un gestor eficiente que se identifica con alguna posición que ocupa una persona dentro o fuera de una organización y también cuenta con ciertas características personales que expresan los líderes (Bolívar, 1997; Hellriegel et al., 2008). Entre ellas está la autoconciencia como la habilidad que se demuestra a la hora de reconocer y comprender las ideas e impulsos de las personas con las que se relaciona. A partir de esta habilidad se podrán organizar las tareas de manera adecuada de acuerdo con los perfiles identificados.

Por otro lado, se señala el autocontrol, habilidad que permite al líder normalizar y regular los impulsos y deseos personales. Los líderes no dejan de ser personas y por lo tanto no quedan exentos de estos sentimientos, por lo que el tratamiento efectivo les permitirá realizar sus actividades sin que se vean afectadas. En tercer lugar, colocan a la conciencia social como la habilidad para realizar una lectura eficiente de las reacciones emocionales de otras personas y sentir empatía con las mismas. Ante la información o sucesos que pueden llegar a suceder, el líder maneja la situación de una manera en que no se afecte a sus seguidores. Por último, establecen a la habilidad social como la capacidad de líder para el manejo de las relaciones sociales dentro del equipo de trabajo. A través de la creación de equipos y/o redes interpersonales, buscan conseguir alcanzar los objetivos planteados al hacer que sus seguidores se muevan en la misma dirección.

Por otro lado, Burns (1978) identificó dos tipos de liderazgos, el transaccional y el transformacional. Plantea que el primero se limita a la relación costo-beneficio, ya que los seguidores solo desempeñan su labor a cambio de un valor. Contrario a esto, el liderazgo transformacional busca que los seguidores se comprometan con la misión del líder o de la organización a la cual pertenecen, dejando en un segundo término las motivaciones e intereses personales. A partir de esta clasificación, Bass (1985) desarrolló el modelo de liderazgo 


\section{Journal of the Academy | $13 \mid$}

transformacional mediante el cual la persona líder logra de manera eficiente conectar con sus seguidores para lograr que se identifiquen con su misión y con sus objetivos y está compuesto por cuatro factores: a) Carisma o influencia idealizada; b) Inspiración o motivación: c) Estimulación intelectual; d) Consideración individual.

De acuerdo con Guest, et al. (2020), son necesarios tres aspectos para acabar con la pandemia provocada por el Covid-19, los cuales son la capacidad de liderazgo de los expertos en el área de la salud pública, las innovaciones científicas que generan información oportuna y un fuerte compromiso y voluntad entre los actores políticos. En este sentido, Antonakis (2021) apunta que los líderes políticos tienen el poder de influir sobre las acciones de los individuos por lo que, en el marco de la mencionada pandemia, necesitan comunicar la información obtenida a partir de evidencias científicas, así como convertirse en una imagen de ejemplo del comportamiento deseado entre los ciudadanos. Así como menciona Bass (1985), Antonakis (2021) también subraya la importancia del carisma entre los líderes políticos como una manera emocional y simbólica de dirigir las acciones de los individuos a partir de la empatía para así unificar y evitar contestaciones.

Para Anwar, et al. (2020) el aislamiento que ocasiona la epidemia genera miedo, ansiedad e incluso otro tipo de enfermedades, esto puede combatirse mediante el uso responsable de los medios de comunicación, al existir el adecuado control y contrapeso de la imagen, confianza y liderazgo que tengan los voceros oficiales para prevenir la propagación de rumores y acabar con la estigmatización de los afectados por el Covid-19, por lo tanto la relación entre medios y gobierno resulta fundamental en estos tiempos.

\section{METODOLOGÍA}

Se realizó un estudio cualitativo a través de la técnica de grupos focales para identificar la percepción ciudadana en el estado de Nuevo León, siendo este uno de los lugares con más población en México con 5784442 habitantes y más de 40000 empleos formales de acuerdo con el Instituto Nacional de Estadística y Geografía (INEGI, 2020). Se considera esta técnica de grupos focales pertinente para recabar datos sobre temas en específico por medio de guiones que vayan llevando a las personas a hablar sobre las categorías que se buscan explicar. De igual forma la literatura (Kitzinger, 1995; Santiago y Roussos, 2010; Hamui-Sutton y Varela-Ruiz, 


\section{Journal of the Academy $|14|$}

2013) aconseja dividir por edad y género en grupos de seis a ocho personas para obtener una mayor validación interna de acuerdo con las categorías que se manejaron: liderazgo, imagen y confianza. Por lo que se generaron cuatro grupos focales de ocho personas, cada uno: dos de jóvenes y dos de adultos separados por género.

Se tomaron también en cuenta las consideraciones del INEGI (2020) donde indica que los jóvenes son las personas de 15 a 29 años y los adultos aquellos de 30 años en adelante. Cabe señalar que estos grupos focales se llevaron a cabo durante el mes de octubre del 2020.

Según varios autores (Kitzinger, 1995; Hernández, et al. 2014; Caballero, 2014) la información mediática recibida se puede procesar y entender de mejor forma si existe un elevado nivel de confianza en las autoridades que la presentan, así como el manejo de una buena imagen pública y un sólido liderazgo, lo cual lleva a una tranquilidad y estabilidad ciudadana (Bass, 1995; Canel, 2001; Fabián, 2011).

Como líderes, se seleccionaron a las figuras representativas del poder que enteraban sobre el Covid-19. Esto se determinó de acuerdo a que tanto el gobierno federal como estatal determinaron colocando, el primero, al titular de la Subsecretaría de Prevención y Promoción de la Salud el Dr. Hugo López-Gatell Ramírez y el segundo al secretario de Salud el Dr. Manuel Enrique de la O Cavazos, quien funge a su vez como director general de los Servicios de Salud de Nuevo León como voceros oficiales de la situación sobre la pandemia. De igual forma tanto el presidente de la República Mexicana Andrés Manuel López Obrador como el gobernador del estado de Nuevo León Jaime Rodríguez Calderón debido a sus investiduras políticas se vuelven voceros nacionales y estatales de lo que acontece con la epidemia.

Dado que esta es una investigación de corte cualitativo, se buscó generar constructos con base en las diferentes categorías que a su vez son precisadas por los distintos ítems en los que se basa el estudio para hacer las preguntas en los grupos focales. Para la imagen los ítems fueron características personales de los voceros (Puig, 2009; Canel, 2001), posicionamiento ideológico (Canel, 2001) y habilidades comunicativas (Chihu, 2010; Gómez, 2001; Canel, 2001); el liderazgo se estudió con base en su posición transformacional (Bass, 1985), emocional (Goleman, 2018) y asertiva (Elizondo, 1999; Delgado, 2004); por último la confianza se generó gracias a los ítems de Credibilidad (Fabián, 2011), eficiencia (Segovia et al., 2008) y transparencia (Martínez, 2016). 


\section{Journal of the Academy | 15 |}

Se realizó una prueba piloto para la validación del instrumento con dos grupos de ocho personas uno de adultos y otro de jóvenes para identificar si las preguntas se entendían, eran repetitivas o causaban conflicto, y de ser así poder adecuarlas. El único apartado que causó dudas fue el posicionamiento ideológico, el cual se definió de acuerdo a lo estudiado por Canel (2001) quien lo explica como los sentimientos políticos que llevan a militar en grupos de interés y la afinidad que expresan los ciudadanos hacia ciertos partidos políticos.

\section{RESULTADOS}

El Covid-19 vino a cambiar la normalidad de la mayoría de los ciudadanos. La forma en que es percibida la pandemia, así como la confianza, imagen y liderazgo de las personas encargadas de informar sobre esta situación se describe a continuación con el propósito de explicar este fenómeno mediante una metodología cualitativa.

La mayoría de los individuos que participaron en el estudio tendían a relacionar la pandemia con el encierro y era de los temas que sin importar género o edad estaban presentes en todos los grupos. En general los cuatro grupos hicieron referencia a esta situación llamándola cuarentena, aislamiento o de otras formas, pero refiriéndose siempre al confinamiento que esta enfermedad trajo, sin importar la edad o el género. Mientras que los adultos hacían mención también a una crisis económica y culpaban al gobierno, los jóvenes hablaban sobre el desempleo y una constante duda sobre la gravedad real de la enfermedad. Los hombres expresaban más esta duda que las mujeres haciendo referencia a que podía ser incluso un distractor o una cortina de humo, por su parte las mujeres se enfocaban más en temas como la convivencia familiar, pero a su vez mencionaban que el trabajo laboral se sentía ahora más pesado que antes y que las empresas no respetaban los horarios.

El medio de comunicación que más utilizaron las personas de los grupos focales para informarse sobre la pandemia fue sin duda internet, haciendo hincapié en las redes sociales, aunque también les preocupaba la cantidad de información falsa que podían consumir. La televisión se convirtió en el medio predominante de los adultos, mientras que los jóvenes hicieron referencia al periódico en formato online, específicamente a la red social Twitter donde veían los tweets de la prensa oficial. El radio fue mencionado por los adultos sobre todo por los hombres, las mujeres adultas preferían la televisión y solo pocas el periódico. Los 


\section{Journal of the Academy | $16 \mid$}

jóvenes sin importar género, decían no ver televisión y solo enfocarse en internet. Solo dos mujeres jóvenes hicieron referencia al radio porque en el trabajo sus compañeras lo ponían. Las personas más relacionadas con el tema de la pandemia fueron por parte de todos: el Dr. Hugo López-Gatell y el Dr. Manuel de la O. En segundo lugar, se menciona al presidente Andrés Manuel López Obrador y al gobernador de Nuevo León, Jaime Rodríguez. El Dr. Jorge Alcocer y el presidente de los Estados Unidos de América Donald Trump fueron señalados por los adultos, mientras que los jóvenes hablaron de Instituciones como el Instituto Mexicano del Seguro Social (IMSS), el Instituto Tecnológico y de Estudios Superiores de Monterrey (ITESM) y la Universidad Autónoma de Nuevo León (UANL). Con base en ello, se concentran las percepciones de imagen, liderazgo y confianza de cada personaje de mayor a menor mención.

\section{Imagen}

La imagen del Dr. López-Gatell Subsecretario de Salud del Gobierno Federal, sobresale por su simpatía. En los hombres destaca por ser agradable y para la mujer es confiable y atractivo. Los jóvenes sin importar el género lo ven simpático, confiable y como alguien educado. Las mujeres lo perciben como elegante. Los hombres jóvenes hacen referencia a que parece alguien que tiene dinero. Los hombres adultos lo relacionan con Morena y creen que puede aspirar a participar de forma más activa en la política. Las mujeres jóvenes resaltaron de nuevo la confianza que sienten al verlo explicando detalles de la enfermedad o incluso haciendo chistes para romper un poco la tensión en las sesiones informativas.

En el caso del Dr. Manuel de la O. la percepción que se tiene sobre su imagen es de una persona enérgica y todos los participantes hicieron referencia a su bata de médico como algo muy característico de él. Para los jóvenes es alguien que regaña y enojón, para los adultos es una persona justa que habla las cosas de forma seria y con nivel de preparación. Los hombres jóvenes lo perciben más como alguien monótono, las mujeres jóvenes hicieron referencia a él como alguien sencillo y educado. Las mujeres adultas lo mostraron como alguien preocupado por el bienestar de la sociedad y los hombres adultos no lo ven como alguien que quiera participar activamente en la política sino más bien como alguien que le gusta su trabajo y busca concientizar a la gente. 


\section{Journal of the Academy | $17 \mid$}

El gobernador Jaime Rodríguez Calderón es retratado como un caudillo. Para las mujeres resulta alguien autoritario y grosero, mientras que los hombres lo ven como alguien impositivo y en ocasiones simpático. Los adultos lo relacionan con el PRI (Partido Revolucionario Institucional) y con ser un hombre de familia. Los jóvenes también como alguien autoritario, pero hicieron mucha referencia al ser ranchero. Las mujeres adultas creen que seguirá por el camino de la política y los hombres adultos lo catalogan más como ignorante. Para las mujeres jóvenes es alguien que representa al machismo y los hombres jóvenes destacaron la seguridad que tiene para expresarse sin importar que esté diciendo algo correcto o equivocado.

Por último, la imagen del presidente Andrés Manuel López Obrador fue la que más dividió opiniones mientras que para casi la mitad resultó simpático para la otra mitad no. En común se habla sobre su aspecto de abuelito, el partido de Morena y su ideología de izquierda, para los jóvenes es alguien más lento y aburrido, mientras que para los adultos puede ser monótono y pausado. Los hombres adultos hicieron referencia a su habilidad política y al socialismo, de nuevo de forma polarizada, las mujeres adultas comentaron que parece seguir en elecciones. Los hombres jóvenes mencionaron que habla mucho de religión, específicamente en temas de Covid-19. Las mujeres jóvenes dijeron que es una figura familiar, es como alguien conocido que sabes que siempre estará en los medios.

\section{Tabla 1}

Atributos de imagen

\begin{tabular}{lllll}
\hline & Hugo López-Gatell & Manuel de la O & Jaime Rdgz. C. & AMLO \\
\hline Atributos & Simpático & Enérgico & Caudillo & Simpático \\
de & Confiable & Profesional & Impositivo & Engreído \\
imagen & Agradable & Enojón & Autoritario & Lento/Hábil \\
\hline
\end{tabular}

Fuente: Elaboración propia, basada en la información obtenida de los grupos focales.

\section{Liderazgo}

El Dr. López-Gatell es considerado de manera general como un líder carismático, para los hombres es un líder nato y para las mujeres es un líder inspiracional, que motiva. Las mujeres jóvenes lo ven como alguien abierto y esperanzador que sobre todo sabe explicar los temas de la pandemia sin reservas y buscando una solución a lo que acontece. Para los hombres jóvenes 


\section{Journal of the Academy $|18|$}

es un líder empático, propositivo y capaz de entender lo que está sucediendo y como les afecta; de forma similar para los hombres adultos López-Gatell es un líder asertivo y que busa beneficiar a todos y hablar de las cosas con un tono elocuente que busca soluciones y no más problemas. Las mujeres adultas en varias ocasiones hicieron referencia a que era un líder inteligente que estimulaba a la creatividad del mexicano para salir de situaciones difíciles que se presentaban con la pandemia. En general y de acuerdo con la teoría representa a un líder transformacional.

Como temerario, pero con carisma fueron las características que se mencionaron de forma general del Dr. Manuel de la O. El género no hizo mucha distinción en la percepción del liderazgo de este personaje, fue más bien la edad la que dividió comentarios, los adultos lo relacionaban mucho con cierres masivos, pero necesarios de algunos negocios, piensan que esta situación provoca un elevado nivel de desempleo que a su vez puede llevar a una crisis económica bastante seria, pero que si no se cumplen sus indicaciones se generaría una crisis sanitaria.

Por su parte los jóvenes hicieron mucha referencia a los recursos audiovisuales que ha usado el Dr. para ejemplificar aspectos del Covid-19 desde videos sacados de YouTube hasta memes, a pesar de esto lo consideran un líder que a ellos no los motiva y que busca que la demás gente acuse o delate fiestas o reuniones clandestinas, lo consideran poco empático. Debido a la explicación teórica y aunque no lo mencionaron de forma directa el Dr. de la O. puede ser un ejemplo de mezcla de líder asertivo, tomando en cuenta la situación y actuando para beneficio general y un líder emocional, por los sentimientos que provoca en los ciudadanos.

En general el concepto de Jaime Rodríguez como líder político y sobre todo en la pandemia es malo, en el inicio de la contingencia los adultos alabaron sus acciones, pero comentan que pasó como su gobierno independiente, a la larga desilusionó. Los hombres jóvenes lo identifican como un líder agresivo y las mujeres jóvenes como alguien poco empático y rígido. Las mujeres adultas si detallaron más la forma en que trató de ayudar a los ciudadanos, aunque también hablaron de un liderazgo rígido. Los hombres adultos lo mencionan como alguien desconfiado. En general los participantes lo catalogaron como un mal líder e hicieron referencia a sus ideas separatistas como algo negativo también únicamente por que él lo propone. 


\section{Journal of the Academy $|19|$}

De nuevo en el caso de Andrés Manuel no es la edad ni el género sino la ideología lo que genera la percepción de su liderazgo. Mientras que para unos es un líder desagradable e impositivo, para otros es un líder carismático y asertivo. Algunos adultos lo ven como un líder que divide y otros como el que motiva al pueblo a ser mejores, porque justamente él es del pueblo. Dentro de los jóvenes también surgió la idea del líder divisor e impositivo, pero también de alguien creativo, empático y sentimental. Debido a las actitudes que provoca en la gente y a como lo siguen o rechazan el presidente podría ser un ejemplo de líder emocional.

\section{Tabla 2}

Atributos de liderazgo

\begin{tabular}{lllll}
\hline & Hugo López-Gatell & Manuel de la O & Jaime Rdgz. C. & AMLO \\
\hline Atributos & Carismático & Asertivo & Mal líder & Carismático \\
de & Inspiracional & Carismático & Agresivo & Desagradable \\
liderazgo & Transformacional & Emocional & Poca empático & Emocional
\end{tabular}

Fuente: Elaboración propia, basada en la información obtenida de los grupos focales.

\section{Confianza}

El último constructo muestra al Dr. Hugo López-Gatell como una persona con trayectoria, sincera y eficiente a pesar de ser alguien joven. Los adultos lo ven como alguien que maneja información actual sobre el Covid-19 y los jóvenes como alguien a quien es fácil entender los temas que trata. Los hombres adultos lo ven como alguien objetivo y que no maneja información falsa. Las mujeres adultas lo perciben como alguien que se maneja con transparencia y que no oculta información. Similar a esto, las mujeres jóvenes lo ven como alguien que explica de forma detallada sus informes y es sincero, mientras que para los hombres jóvenes es alguien que no aburre cuando habla, genera contenido ameno y entendible para todos. Por lo tanto, el nivel de confianza del que goza este personaje es bastante elevado.

El Dr. de la O resulta para la mayoría confiable, aunque, los jóvenes lo consideran exagerado y que maneja demasiada información lo cual en ocasiones es difícil de entender. Los adultos consideran que es alguien experto y con la madurez necesaria para saber lo que hace, aunque esto les pueda traer conflictos económicos en un futuro debido a la contingencia. Las mujeres 


\section{Journal of the Academy | 20 |}

adultas lo definen como alguien elocuente y los hombres adultos como alguien sincero. Las mujeres jóvenes consideran que su manera de informar en ocasiones es lenta y repetitiva y los jóvenes cuestionan la agresividad y el enojo con el que en ocasiones se expresa.

La desacreditación marca la forma en que es percibido el Gobernador de Nuevo León. Los adultos lo ven como alguien falso y los jóvenes como una persona que los engañó. Es percibido como alguien con muy mala comunicación por parte de los hombres adultos y como alguien que ilusionó y no cumplió por las mujeres adultas. Las mujeres jóvenes no creen en su sinceridad y hablan de poca transparencia en su mandato, mientras que los hombres jóvenes lo ven como alguien corrupto lo que genera muy poca confianza en la embestidura gubernamental.

En el caso de Andrés Manuel se presenta la duda de que la mafia del poder lo deje gobernar, pero también la sospecha de que sea digno de confianza. Mientras que para los adultos existe de igual forma una confianza en su experiencia, aparece también un descontento. Para los jóvenes su experiencia y madurez contrastan con la idea de una persona problemática. En el caso de los hombres adultos se trató mucho el arrepentimiento de este personaje y el haber aprendido de situaciones pasadas. Las mujeres adultas hablaron también sobre la subjetividad que en ocasiones maneja para ciertos temas lo cual genera cierto descontrol en su gobernabilidad, tema que retoman los jóvenes, definiendo que, sí existe un grave problema entre el Estado y actores no estatales, en ocasiones culpando al presidente y en otras indicando que todo es parte de un complot.

\section{Tabla 3}

Atributos de confianza

\begin{tabular}{lllll}
\hline & Hugo López-Gatell & Manuel de la O & Jaime Rdgz. C. & AMLO \\
\hline Atributos & Con trayectoria & Confiable & Desacreditado & Causa duda \\
de & Eficiente & Exagerado & Engañoso & Confiable \\
confianza & Sincero/Joven & Madurez & Falso & Maduro \\
\hline
\end{tabular}

Fuente: Elaboración propia, basada en la información obtenida de los grupos focales. 


\section{Journal of the Academy $|21|$}

\section{Discusión de resultados}

El estudio indicó que las personas consideran a la pandemia como un encierro y como una situación de crisis por diferentes motivos como las pérdidas económicas, el desempleo y la gravedad real de la enfermedad. También se observó que los personajes políticos que más identifican los ciudadanos con este tema son el vocero federal, el Dr. Hugo López-Gatell, y el vocero estatal el Dr. Manuel de la O. En segundo término, se mencionaron a los ejecutivos de las mismas demarcaciones, el presidente Andrés Manuel López Obrador y el gobernador Jaime Rodríguez Calderón. Esto concuerda por lo expuesto por en el apartado teórico en donde se mencionó que ante situaciones de crisis es necesario que se presente un vocero que se encargue de comunicar y orientar a la población durante dichas situaciones, con una imagen que de confianza y seguridad a la sociedad (PAHO, 2010).

El Dr. Hugo López-Gatell fue considerado como una persona simpática, agradable, confiable, atractiva y elegante. De acuerdo con Canel (2001), se puede afirmar que el vocero de la pandemia por Covid-19 del gobierno federal tiene una buena imagen ante las personas, ya que sus características personales, como la personalidad, la vestimenta y aspectos físicos son bien recibidas. Por otro lado, el Dr. De la O se destacó por ser asertivo, confiable, así como por su profesionalismo y vestimenta (Canel, 2001; Chihu, 2010, Gómez, 2001) ya que las personas mencionaron que se recuerdan al vocero estatal de la pandemia como una persona enérgica, madura y que siempre trae puesta su bata. Por parte del presidente López Obrador, la mitad de las personas lo describieron como simpático y engreído, que les recordaba a un abuelito, ya que toca bastantes temas familiares. De acuerdo con la clasificación de Canel (2001), la personalidad de este político genera controversia y dos formas muy distintas de percepción por parte de las personas, esto en parte también por la imagen tan fuerte de su ideología política y la cercanía con su partido político. En cuanto al gobernador Jaime Rodríguez, fue descrito como un caudillo y una persona impositiva y autoritaria. Estos elementos descritos quedan fuera de lo que se considera una imagen positiva de los políticos en cuanto a las características personales y el posicionamiento ideológico que percibe la ciudadanía.

Respecto al liderazgo que proyectan estos cuatro personajes políticos, las personas perciben al Dr. López-Gatell como un líder carismático, nato, inspiracional e inteligente. De acuerdo con Bass (1985) el perfil de este personaje sería el de un líder transformacional que, a través de su 


\section{Journal of the Academy | $22 \mid$}

carisma e inspiración, logra conectar con la ciudadanía, si bien se recogieron opiniones que lo consideran empático y asertivo, sus niveles no fueron tan elevados en comparación a la de la percepción de un líder transformacional. El Dr. De la O también se destaca por su carisma, aunque también se considera un líder temerario y acusador, se mencionó su labor durante la crisis en la que no motiva a las personas, pues sus acciones como los cierres masivos no fueron bien vistos. De acuerdo con Bass (1985) y Goleman (2018) es un líder poco emocional, con bajos niveles de inspiración a la ciudadanía, aunque con niveles interesantes de carisma, aunque del líder transformacional sea el único elemento en el que destaque.

El gobernador Rodríguez Calderón también es considerado como un mal líder, al ser agresivo y que demuestra poca empatía a la ciudadanía. Es percibido como todo lo contrario a un líder transformacional (Bass, 1985) ya que no parece ser un buen ejemplo para la ciudadanía por las menciones a su nivel de rigidez y desconfianza. De igual manera que la imagen, el liderazgo percibido por los ciudadanos sobre López Obrador genera controversia entre las personas que participaron en el estudio, ya que por un lado es considerado desagradable e impositivo y, por otro lado, carismático y motivador.

Por último, en cuanto la confianza que generan en los espectadores, el Dr. López-Gatell es considerado como una persona con trayectoria importante y joven, lo que le genera alto nivel de credibilidad (Fabián, 2011), también un político eficiente que es capaz de resolver problemas (Segovia et al., 2008) y transparente al dar la información con sinceridad (Martínez, 2016). El Dr. De la O fue considerado como una persona confiable, aunque un tanto exagerado, se destaca por su madurez y elocuencia al dar información. Se hablaría por lo tanto de que estas características generan confianza por su credibilidad y eficiencia (Fabián, 2011; Segovia et al., 2008), aunque respecto a la transparencia, la percepción es negativa se denota poca sinceridad de lo que menciona (Martínez, 2016).

De nueva cuenta, la percepción del gobernador, en cuanto a confianza, es negativa. Consideran que es un servidor público desacreditado, que engaña y es falso. De la misma manera su forma de comunicarse no es la adecuado, de esta manera sus niveles de credibilidad, eficiencia y transparencia hacen que sea considerado como un servidor público poco confiable (Fabián, 2011; Segovia et al., 2008; Martínez, 2016). La confianza en el presidente, de acuerdo con los datos presentados, es percibida como baja. Existe una gran duda en cuanto a la sinceridad con 


\section{Journal of the Academy $\mid 23$ |}

la que habla a la ciudadanía, así como la información y/o datos que proporciona. De la misma manera que las características anteriores, esta figura pública divide en dos grandes grupos a las personas, los que lo perciben positivamente y los que lo se hacen de manera negativa.

\section{CONCLUSIONES}

Como se ha expuesto previamente, la mayoría de las y los participantes expresaron sentimientos de encierro y de aislamiento causados por el confinamiento decretado por las autoridades como una medida para contrarrestar el avance de la pandemia. En este sentido, las emociones expresadas fueron contrarrestadas por la imagen positiva y confianza depositada en el subsecretario Hugo López-Gatell quien fue percibido como un líder nato, carismático y transformacional, además de ser considerado como un miembro de la clase privilegiada. Por otro lado, el resto de los actores políticos fueron percibidos como poco empáticos, como los representantes de Nuevo León, o bien generaron opiniones polarizadas como en el caso del presidente Andrés Manuel López Obrador.

Cabe señalar que, así como sostienen Burns (1978), Miller et al. (1986), Martín-Salgado (2002) y Antonakis (2021), el carisma es un factor sustancial para generar empatía con la imagen de un actor político, aunque no necesariamente se relaciona con la confianza hacia el liderazgo del mismo. De esta manera, el Dr. de la O Cavazos se describe como un personaje carismático pero que genera desconfianza pues pone en riesgo la economía del estado y fomenta la enemistad entre ciudadanos ya que invita a denunciar a aquellos que incumplen con las medidas establecidas por la autoridad que él encabeza. Por su parte, también se resalta el carisma de López Obrador, sin embargo, se pone en duda la confianza hacia él ya que se percibe como alguien engreído, que polariza a la sociedad a través de su discurso ideológico.

En general, los ciudadanos orientan sus acciones y percepciones sobre la pandemia a través de la información trasmitida a través de los medios de comunicación, sin embargo, se observa una diferencia entre los medios a los que acuden los jóvenes y los adultos. De esta forma, el grupo de los adultos, tanto hombres como mujeres, prefieren informarse por medio de la televisión, dando continuidad a la idea propuesta por Canel en el 2006, mientras que los jóvenes se alejan 


\section{Journal of the Academy | $24 \mid$}

de este medio, el cual incluso ignoran, y confían en la información proporcionada a través de internet, en específico de Twitter, aunque la fuente original es la prensa, lo cual refiere a una digitalización de este medio tradicional.

Por último, cabe recordar que estos grupos focales fueron realizados en octubre del 2020, siete meses después de que la Organización Mundial de la Salud declaró como pandemia el contagio provocado por el Covid-19, la cual sigue vigente aún en junio del 2021 por lo que la imagen, la confianza y el liderazgo de los personajes analizados podrían haber sufrido cambios, siendo interesante darle continuidad a este tipo de estudios para comparar el desarrollo que se ha ido dando.

\section{REFERENCIAS BIBLIOGRÁFICAS}

Antonakis, J. (2021). Leadership to defeat COVID-19. Group Processes \& Intergroup Relations (GPIR), 24(2), 210-2015. https://doi.org/10.1177/1368430220981418

Anwar, A., Malik, M. \& Raees, V. (2020) Role of Mass Media and Public Health Communications in the COVID-19 Pandemic. Cureus, 12(9), 1-12. https://dx.doi.org/10.7759/cureus.10453

Bass, B. (1985) Leadership and Performance Beyond Expectations. The Free Press.

Benedicto, J. y Morán, M. L. (2014). ¿Otra clase de politización? Representaciones de la vida colectiva y procesos de implicación cívica de los jóvenes en situación de desventaja. $\begin{array}{llll}\text { Revista Internacional de } & \text { Sociología, }\end{array}$ https://doi.org/10.3989/ris.2013.02.21

Beramendi, M., Delfino, G., y Zubieta, E. (2016). Confianza Institucional y Social: Una Relación Insoslayable. Acta de Investigación Psicológica - Psychological Research Records, 6(1), 2286-2301. https://doi.org/10.1016/s2007-4719(16)30050-3

Bolívar, A. (1997). Liderazgo, mejora y centros educativos. En A. Medina (Coord.), El liderazgo en educación (pp. 25-46). UNED.

Burns, J. (1978). Transforming Leadership. Grove Press.

Caballero, A. (2014). Metodología integral innovadora. Cengage Learning.

Califano, B. (2015). Los medios de comunicación, las noticias y su influencia sobre el sistema político. Revista mexicana de Opinión Pública, 2, 61-78. http://dx.doi.org/10.1016/j.rmop.2015.02.001

Canel, M. (2001). Comunicación política. Técnicas y estrategias para la sociedad de la información. Tecnos. 
Canel, M. (2006). Comunicación Política. Una guía para su estudio y práctica. Tecnos.

Chihu, A. (2010). El framing audiovisual del spot político. Cultura y representaciones sociales, 5(9), 174-197.

Delgado, S. (2004). Sobre el concepto y el estudio del liderazgo político. Una propuesta de síntesis. Psicología Política, 29, 7-29.

Elizondo, M. (1999). Asertividad y escucha activa en el ámbito académico. Editorial Trillas.

Fabián, R. (2011). Los jóvenes ante la política. Un panorama sobre la percepción del ámbito político. Revista Mexicana de Opinión Pública, (10), 187-199. http://dx.doi.org/10.22201/fcpys.24484911e.2011.10.41783

Glik D.C. (2007). Risk communication for public health emergencies. Annual Reviews of Public Health, 28, 33-54. http://doi.org/10.1146/annurev.publhealth.28.021406.144123

Goleman, D. (2018). Inteligencia emocional en la empresa. Ediciones B.

Gómez, R. (2001). Análisis de la imagen: estética audiovisual. Ediciones Laberinto.

Güemes, C. (2016). Trátame suavemente. Confianza social en Latinoamérica; Argentina bajo la lupa. FLACSO.

Guest, J. L., del Rio, C., y Sanchez, T. (2020). The Three Steps Needed to End the COVID-19 Pandemic: Bold Public Health Leadership, Rapid Innovations, and Courageous Political Will. JMIR Public Health Surveill, 6(2), 8-11. https://doi.org/10.2196/19043

Hamui-Sutton, A. y Varela-Ruiz, M. (2013). La técnica de grupos focales. Investigación en educación médica, 2(5), 55-60. https://doi.org/10.1016/S2007-5057(13)72683-8

Hellriegel, D., Jackson, S. y Slocum Jr., J. (2008). Administración. Un enfoque basado en competencias. Cengage Learning.

Hernández, R., Fernández, C. y Baptista, P. (2014). Metodología de la Investigación. Mcgraw Hill.

Hiskey, J. y Seligson, M. (2003). Pitfalls of Power to the People: Decentralization, Local Government Performance, and System Support in Bolivia. Studies in Comparative International Development, 37(4), 64-88. https://doi.org/10.1007/BF02686272

Instituto Nacional de Estadística y Geografía. (2020). Censos y conteos de población y vivienda. https://www.inegi.org.mx/programas/ccpv/2020/

Kitzinger J.(1995) Qualitative Research: introducing focus group. BMJ, 311, 299-302. https://doi.org/10.1136/bmj.311.7000.299

Kleinberg, B., Van der Vegt, I. y Mozez, M. (2020). Measuring emotions in the covid-19 real world worry dataset. En K. Verspoor, K. Bretonnel Cohen, M. Dredze, E. Ferrara, J. 


\section{Journal of the Academy | $26 \mid$}

May, R. Munro, C. Paris, y B. Wallace (Eds.), Proceedings of the 1st workshop on NLP for COVID-19 at ACL 2020. Association for Computational Linguistics. https://www.aclweb.org/anthology/2020.nlpcovid19-acl.11

Landau, M. (2009). La conflictiva relación entre participación institucionalizada y confianza: el caso de Buenos Aires. Perfiles latinoamericanos, 17(34), 111-124.

Malecki, K., Keating, J. y Safdar, N. (2020). Crisis communication and public perception of COVID-19 risk in the Era of Social Media. Clinical Infectious Diseases, 72(4), 697-702. https://doi.org/10.1093/cid/ciaa758

Martín-Salgado, L. (2002). Marketing Político. Arte y ciencia de la persuasión en democracia. Paidós Ibérica.

Martínez, J. (2016). Transparencia y derecho a la información pública en México. Avances, retos y perspectivas. El Cotidiano, 198, 14-26

Miller, A., Martin, P. y Oksana, M. (1986). Schematic assessments of presidential candidates. American Political Science Review, 80(2), 521-540. https://doi.org/10.2307/1958272

Montaña, M., Ollé, C. y Lavilla, M. (2020). Impacto de la pandemia de Covid-19 en el consumo de medios en España. Revista Latina de Comunicación Social, 78, 155-167. https://www.doi.org/10.4185/RLCS-2020-1472

Orejuela, S. (2009). Personalización política: la imagen del político como estrategia electoral. Revista de Comunicación, 8, 60-83.

Pan American Health Organization. (2010). Comunicaciones sobre riesgos durante crisis y situaciones de emergencia. OMS.

Puig, J. M. (2009). Aprendizaje servicio (APS): educación y compromiso cívico. Graó.

Rodero, E. (2020). "La radio: el medio que mejor se comporta en las crisis. Hábitos de escucha, consumo y percepción de los oyentes de radio durante el confinamiento por el Covid19”. El profesional de la información, 29(3), 114. https://doi.org/10.3145/epi.2020.may.06

Santiago, J., y Roussos, A. (2010). El focus group como técnica de investigación cualitativa. Documento de trabajo $\mathrm{N}^{\mathrm{O}} 256$. Universidad de Belgrano. http://repositorio.ub.edu.ar/handle/123456789/4781

Segovia, C., González, R., Haye, A. y Manzi, J. (2008). Confianza en instituciones políticas en Chile: un modelo de los componentes centrales de juicios de confianza. Revista de Ciencia Política, 28(2), 39-60. http://dx.doi.org/10.4067/S0718-090X2008000200002 